\title{
Structural Snapshots of a Dynamic Process in the Regulation of P-Rex, a Metastatic Factor
}

\author{
Jennifer N. Cash ${ }^{1}$, Sandeep Ravala ${ }^{2}$, John J.G. Tesmer ${ }^{2}$, and Michael A. Cianfrocco ${ }^{1}$ \\ 1. Life Sciences Institute, Department of Biological Chemistry, University of Michigan, Ann Arbor, MI, \\ United States \\ 2. Department of Biological Sciences, Purdue University, West Lafayette, IN, United States
}

Cell migration is a complex cellular task that involves the coordinated activity of hundreds of intracellular components. Near the top of this signaling pyramid is P-Rex (Phosphatidylinositol 3,4,5trisphosphate-dependent Rac exchanger), a guanine nucleotide exchange factor that activates GTPases responsible for remodeling the actin cytoskeleton in response to signalling from upstream $G$ proteincoupled receptors (GPCRs) and receptor tyrosine kinases (RTKs) [1]. In order to understand the regulation and activity of P-Rex, we have determined cryo-electron microscopy (cryo-EM) structures of P-Rex bound to allosteric regulators along with substrate. We then combined these structures with previously determined X-ray crystallography structures of subdomains and hydrogen/deuterium exchange mass spectrometry (HDX-MS) to understand the structural changes that accompany P-Rex activation. This work has revealed how P-Rex is capable of integrating signals from both receptor families into its GEF activity, establishing a new model for P-Rex regulation.

In the process of directing cellular movement in response to extracellular cues, signals must be received at the plasma membrane and then amplified within the cytoplasm to direct the remodeling of the cytoskeleton [2]. These signalling events can begin with the activation of GPCRs, which results in the release of $\mathrm{G}$ protein subunits $\mathrm{G} \beta$ and $\mathrm{G} \gamma$ from the GPCR complex. In tandem to this, signaling through RTKs leads to activation of Phosphoinositide 3-kinase (PI3K), generating the signalling lipid $\mathrm{PIP}_{3}$ [3].

P-Rex acts as a signal integrator by combining input from these two signalling pathways. It has been demonstrated that G $\beta \gamma$ and PIP3 synergistically activate P-Rex and recruit it to the cell membrane, likely through an allosteric mechanism [6]. However, the molecular details of this activation are poorly understood. By interacting with both G $\beta \gamma$ and PIP3, P-Rex combines the contributions of these allosteric regulators into GEF activity for Rho-GTPases to potentiate actin cytoskeleton reorganization. This critical role played by P-Rex in GPCR signalling has only become appreciated recently, as P-Rex was only discovered 16 years ago [4]. Given the essential role of P-Rex in directing actin cytoskeleton remodeling for cell migration, P-Rex has become a target to block cancer metastasis. For example, a recent study of found that 11 out of 25 sequenced metastatic melanomas contain an isoform of P-Rex, PRex2, with non-synonymous mutations [5]. Therefore, understanding the mechanism and structural underpinnings of P-Rex activity is critical to understanding the basic function of cell migration in addition to the development of future therapeutics.

In order to study the structural basis for P-Rex to perform these functions, we have combined X-ray crystallography and HDX-MS with single particle cryo-EM to study the allosteric regulation of P-Rex. Our crystallographic studies of a subdomain of P-Rex with substrates cryo-EM structure highlighted how the two domains comprising Dbl homology (DH) and pleckstrin homology (PH) interact with IP4, a soluble PIP3 analog [6]. We then mapped these structures onto HDX data as it relates to P-Rex binding, 
allowing us to interpret our cryo-EM structures within the context of atomic resolution structures and structural dynamics.

Using these structures and data derived from HDX-MS and SAXS experiments, we have developed a new model for how P-Rex activity is regulated. This model helps to describe how allosteric control of PRex is achieved in order to stimulate its GEF activity.

References:

1. D Spiering and L Hodgson, Cell Adh Migr 5 (2011), p. 170.

2. $\quad$ ML Gardel et al, Annu Rev Cell Dev Biol 26 (2010), p. 315.

3. E Wertheimer et al, Cell Signal 24 (2012), p. 353.

4. HCE Welch et al, Cell 108 (2002), p. 809.

5. MF Berger, et al. Nature 485 (2012), p. 502.

6. JN Cash, EM Davis, JJG Tesmer, Structure 24 (2016), p. 730 .

7. This work is supported by an American Cancer Society - Michigan Cancer Research Fund Postdoctoral Fellowship (PF-14-224-01-DMC) to J.C., NIH Grants HL071818 and HL122416 to J.T, and NIH Grant CA221289 to J.T. and M.C. 